\title{
The Expression of Progesterone Receptors in Meningiomas of Different Grades
}

\author{
Mohammad Tahir ${ }^{1}$, Tehreem Atif ${ }^{2}$, Summaya Sohail ${ }^{3}$, Arfa Nawazish ${ }^{4}$, Huma Mushtaq ${ }^{5}$ \\ ${ }^{1}$ Associate Professor, Department of Pathology, Rawal Institute of Health Sciences, Islamabad \\ 2 Specialty Doctor (Histopathology), Glan Clywd Hospital.Wales.UK \\ ${ }^{3}$ MD Resident (Histopathology), Pakistan Institute of Medical Sciences, Islamabad \\ ${ }^{4}$ Specialty Doctor, Anatomical Pathology, Australian Clinical labs, Perth, Australia \\ ${ }^{5}$ Associate Professor, Department of Pathology, Islamabad Medical \& Dental College
}

\section{ABSTRACT}

Background: Meningiomas are slow growing intracranial and intraspinal neoplasms with a tendency to recur locally. WHO grades them as I (benign), II (atypical) and III (anaplastic) in order of their increasing aggressiveness, based on histological parameters and brain parenchymal invasion. Progesterone receptors (PR) are more prevalent amongst the lower grade meningiomas. The objective of this study was to determine the immunohistochemical expression of progesterone receptors in meningiomas of different grades.

Material and Methods: A total of 100 cases were selected over a period of 2.5 years. Three to five microns' thick sections stained with Hematoxylin and Eosin were examined microscopically by a team of two Histopathologists and graded into grades I, II and III, according to 2016 WHO classification criteria. Another section of the original tumor was stained with progesterone receptor antibody using the conventional immunoperoxidase method. Stained slides were than examined by the same team of Histopathologists and declared positive (if nuclear staining was observed in more than $10 \%$ of tumor cells) or negative. Statistical analysis was done using SPSS version 21.

Results: Out of a total of 100 cases of meningioma, there were 79 cases of benign/typical WHO grade I, 15 cases of atypical/ WHO grade II and 6 cases of anaplastic/ WHO grade III tumor. PR status was positive in $89.8 \%$ (71/79) of grade I meningiomas and 46.6 $\%(7 / 15)$ of grade II/Atypical meningiomas. The 06 cases of Anaplastic/WHO grade III tumors were negative for PR. There was a higher prevalence of Progesterone receptors in female patients $(89.8 \% ; 53 / 59)$ as compared to male meningioma patients $(60.9 \%$; 25/41).

Conclusion: We observed a decreased expression of progesterone receptor in higher grades of meningioma in this study. It is an effort to explore conservative treatment options for inoperable lesions, as anti-progesterone therapy may hold a promise as a new treatment option in the near future.

Key words: Meningioma, Immunohistochemistry, Progesterone receptor

Authors' Contribution:

1,2 Conception, synthesis, planning of

research and manuscript writing ${ }^{3}$

Interpretation, discussion, ${ }^{4,5}$ Active

participations in data collection

Data analysis.

Cite this article. Tahir M, Atif T, Sohail S, Nawazish A, Mushtaq H. The Expression of

Progesterone Receptors in Meningiomas of Different Grades. J Islamabad Med Dental Coll.2019; 8(2):65--69

\section{Introduction}

Meningiomas are benign, slow growing, locally recurring intracranial and intraspinal neoplasms that are known to have sex steroid receptors expression. ${ }^{1-3}$ The discovery of such receptors was based on the association of meningiomas with female gender, breast cancers ${ }^{4}$, pregnancy related growth and luteal phase of menstrual cycle. ${ }^{5}$ Progesterone receptors status and proliferation indices are known prognostic indicators of meningiomas. ${ }^{6}$ 
Several studies indicate progesterone receptor correlation with gender, age as well as WHO grades (I, II and III). ${ }^{4}$

Regarding sex hormone receptors expressed in meningiomas, about $70 \%$ are progesterone receptors, ${ }^{7}$ primarily associated with lower grade meningiomas. An Indian study assessing the PR status in different grades of meningiomas observed that $70 \%$ of grade I meningiomas in contrast to only $20 \%$ Grade II meningiomas, were PR Positive. There was a single grade III meningioma case in this study, which was reported as PR negative. ${ }^{6}$ Similarly recurrent meningiomas were characterized by a consistent negativity for progesterone receptors. ${ }^{8}$ Variable progesterone receptor expression is frequently seen in atypical group of meningiomas. ${ }^{9}$ The role of hormones, specifically progesterone, in pathogenesis of meningiomas is further highlighted by the two-fold greater occurrence of meningiomas in females, the hormonal status of female patients and the use of exogenous hormones as contraceptive pills (OC). ${ }^{10,11}$

Although underestimated in the past, introduction of novel chemotherapeutic options suggests that treatment with anti-progesterone receptor agents may prove fruitful for selected patients. ${ }^{12}$ This study was conducted to demonstrate the occurrence of progesterone receptors in meningiomas as there is a limited number of studies available in our part of the world.

\section{Material and Methods}

This study was conducted in the Histopathology Department of Federal Government Polyclinic, PGMI, Islamabad from 1st January, 2013 to 30th June, 2015. It was a cross-sectional study with a sample size of 100 , which was calculated by WHO sample size calculator. Consecutive non- probability sampling technique was employed including all cases reported as Meningiomas of any grade. Cases of both genders in age range of 25 to 70 years were included. However, stereotactic biopsies, tissue exhibiting fixation artifacts and inadequate scant tissue were excluded. Freshly cut, three to five microns' thick sections, stained with Hematoxylin and Eosin $(\mathrm{H}$ \& E) were examined microscopically by a team of two Histopathologists (a Consultant and a Resident
Histopathologist). All cases were graded according to WHO grading system. Another 6 microns thick section of the original tumor were stained with progesterone receptor antibody (anti-PR monoclonal antibodies purchased from DAKO) using the conventional immunoperoxidase method. Benign breast tissue was used as positive controls. Stained slides were than examined by the same team of Histopathologists and declared positive (if nuclear staining was observed in more than $10 \%$ of tumor cells) or negative for PR expression. ${ }^{11}$

The data was entered in SPSS version 21. Frequency and percentages were used to calculate qualitative variables like presence or absence of progesterone receptors by use of immunohistochemistry in meningiomas and its grades. Mean and standard deviations were used to calculate quantitative variables like age of the patient. Tumor areas were first scanned on low-power for maximally stained regions and the stained nuclei were then counted at high-power magnification (40X).

\section{Results}

A total of 100 patients, with the diagnosis of meningioma were included, in this study. Mean age of the patients was $50.33 \pm 12$ years. Female predominance was observed with $59 \%$ females $(n=59)$ and $41 \%$ males $(n=41)$. According to WHO criteria, of all the subtypes of meningioma, the most commonly found entity was benign/typical WHO grade I tumors ( $\mathrm{n}=79$ cases), followed by atypical/grade II tumors ( $n=15$ cases) and anaplastic/grade III tumors ( $\mathrm{n}=6$ cases), respectively. Brain invasion was found in $3 \%$ of cases and none of the cases were recurrent tumors.

Overall the PR status was positive in $78 \%(n=78)$ of meningioma cases in this study, while PR expression was negative in all the three cases showing brain invasion. PR status was positive in $89.8 \%(71 / 79)$ of grade I meningiomas and $46.6 \%(7 / 15)$ of grade II/Atypical meningiomas. The 06 cases of Anaplastic/WHO grade III tumors were negative for PR (Figure 1). There was higher prevalence of Progesterone receptors in female patients 
$(89.8 \% ; 53 / 59)$ as compared to male meningioma patients $(60.9 \% ; 25 / 41)$ as shown in Figure 2.

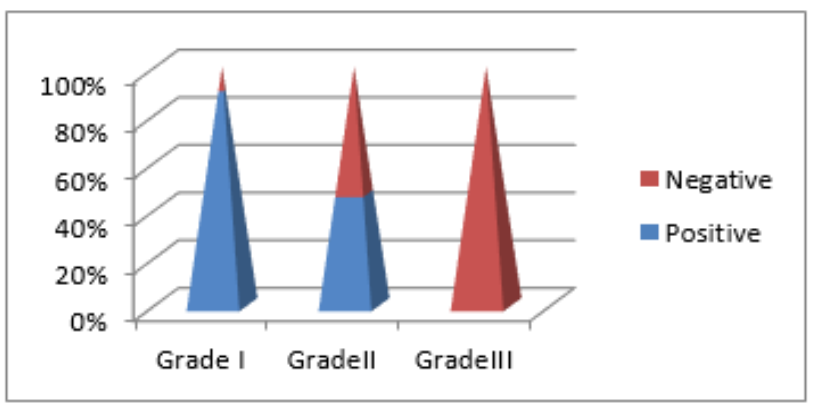

Figure 1: Decreasing trend of PR expression in increasing grades of meningioma

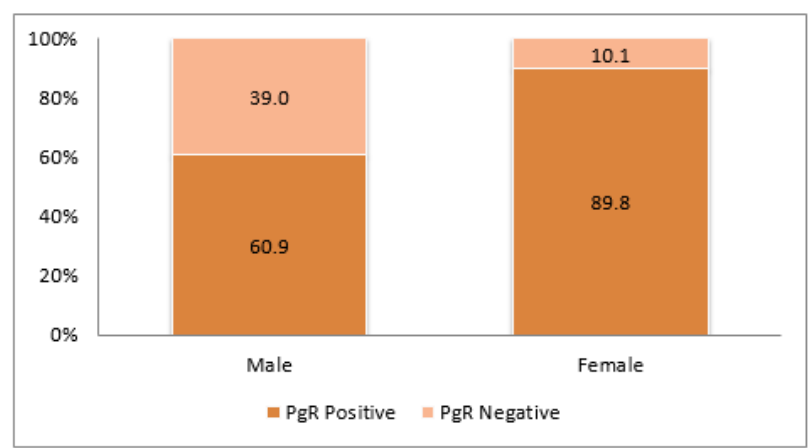

Figure 2: Gender distribution of PR positive and PR negative cases of meningioma.

H\&E stained tissue section of meningioma grade I along with Progesterone receptor strong nuclear positivity is shown in Figure 3. H\&E stain of anaplastic meningioma grade -III, showing loss of whorling pattern, nuclear atypia, nuclear pleomorphism and high mitotic activity is shown in Figure 4, while Progesterone receptor negative staining is shown in Figure 5.

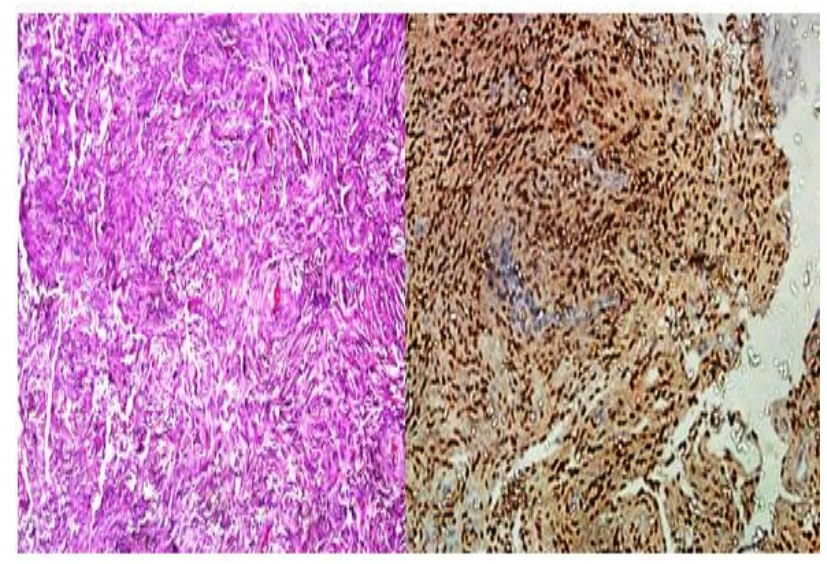

Figure 3: Meningioma grade I showing meningothelial cells arranged in whorling/lobular pattern. PR IHC applied on the same case shows strong nuclear positivity.

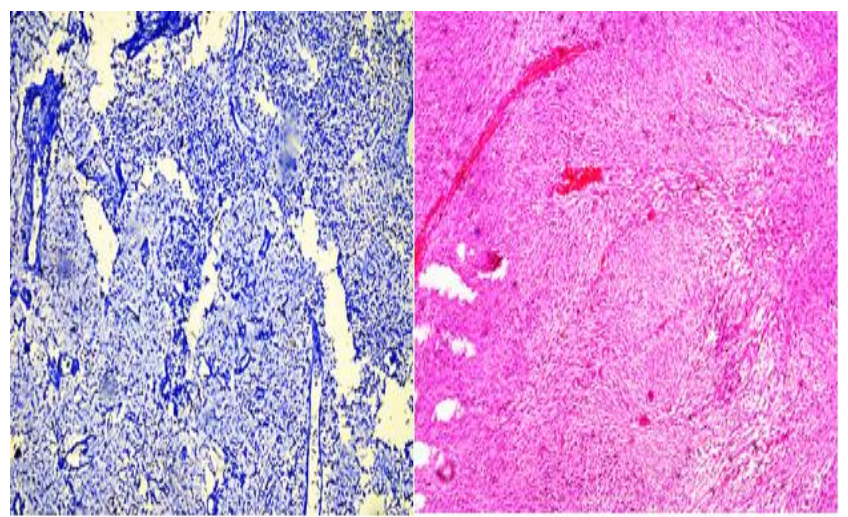

Figure 4: H\&E stain of anaplastic meningioma grade-III, showing loss of whorling pattern, nuclear atypia \& pleomorphism.

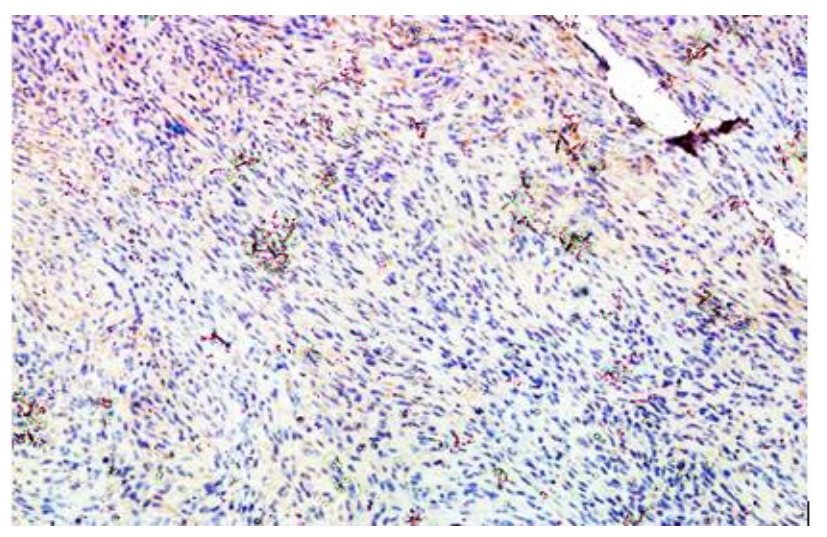

Figure 5: Progesterone receptor IHC stain applied on the above case shows no nuclear staining with adequate controls applied.

\section{Discussion}

Meningiomas account for $20-32 \%$ of all the primary intracranial tumors. According to the WHO 2016 classification system, meningiomas are classified into 3 histological grades and 15 subtypes. This histopathological classification is generally used to predict the clinical course of meningioma. ${ }^{13}$ Even after complete removal, WHO grade I meningiomas frequently recur. The role of steroid hormones in the progression of meningiomas is still a matter of controversy. Our study aims to highlight the expression of PR in different grades of meningiomas. The results establish that immunodetection of progesterone receptors is inversely proportional to the histological grade of the tumor. Hence this can be used for assessing the biological behavior of meningiomas. Statistics of this study are similar to several other studies, which concluded a more favorable prognosis for PR positive meningiomas. ${ }^{10,11}$ 
Gender predilection for females in case of meningioma is evident in most of the studies available in the literature. In this study $59 \%$ of the patients were female while $41 \%$ were males. In a descriptive study conducted in France Nagashima and colleagues collected 13,038 cases of meningiomas. Among these 9769 (74.9\%) were females and $3269(25.1 \%)$ were males. This is in accordance with previously published data. ${ }^{14}$ According to the World Health Organization (WHO) classification, meningiomas are classified into three grades; grade I, II and III as already described. Studies showed that $90 \%$ of all meningiomas are benign tumors and $10 \%$ are in grade II or III, with unfavorable clinical courses. This synchronizes with our data and several other studies. ${ }^{15}$

We observed that PR expression was higher in grade I tumors than grade II and III tumors. These findings are also in concordance to published literature. Similar findings were noted by Iplikcioglu and colleagues who found that PR expression was higher in WHO grade I meningiomas than grade $\mathrm{II}$ and $\mathrm{III} .^{16}$ Cahill et al and Brandis et al showed that malignant meningiomas are devoid of PR and estrogen receptors. Furthermore, Whittle et al reported that PR negative meningiomas were biologically more aggressive than $\mathrm{PR}$ positive meningiomas. ${ }^{17}$ Our data confirms the presence of significantly higher PR values in benign meningiomas compared with WHO grade II or III tumors. The PR expression exhibits marked heterogenicity within the same tumor, owing to the various innovative surgical procedures applied as well as intrinsic heterogeneous nature of the tumor itself. ${ }^{10}$

In addition, our study observed that PR expression was more in female gender than males. In $89.8 \%$ of female patients, Progesterone receptors were found in meningiomas while it was present in $60.9 \%$ of male patients. Perrot-Applanat and colleagues also found similar findings that progesterone receptors were immunostained in $79 \%$ of female patients in contrast to $58 \%$ of male patients. ${ }^{18}$ Mukhopadhyay studied the PR expression along with ER and $\mathrm{Ki}-67$ and found a reciprocal expression of $\mathrm{PR}$ and $\mathrm{ER}$ i.e. higher the grade of the tumor the more is the immunoreactivity for ER but PR expression was lost with higher grade. ${ }^{19}$
One limitation of our study is that we were unable to follow up the patients to see the behavior of the tumor in vivo due to time constraints.

\section{Conclusion}

Our study is a step towards better understanding of pathophysiology of meningioma. It supports the conservative management of meningioma through noninvasive hormonal therapy instead of surgery, which has its own complications. However, more work needs to be done, incorporating other clinical and histopathological parameters as well, so that newer treatment option of anti-progesterone therapy can be further explored.

\section{References}

1. Strassner C, Buhl R, Mehdorn HM. Recurrence of intracranial meningiomas: did better methods of diagnosis and surgical treatment change the outcome in the last 30 years? Neurological Res. 2009; 31(5): 478-82. doi: 10.1179/174313208X338043

2. Shayanfar $N$, Mashayekh $M$, Mohammadpour $M$. Expression of progestrone receptor and proliferative marker Ki 67 in various grades of meningioma. Acta Medica Iranica.2010; 48(3): 142-7. PMID: 21137648

3. Claus EB, Park PJ, Carroll R, Chan J, Black PM. Specific genes expressed in association with progesterone receptors in meningioma. Cancer Res. 2008;68(1):314-22. doi: 10.1158/0008-5472.CAN-07-1796

4. Rao G, Giordano SH, Liu J, McCutcheon IE. The association of breast cancer and meningioma in men and women. Neurosurgery. 2009; 65(3):483-9. doi: 10.1227/01.NEU.0000350876. 91495.E0

5. Hatiboglu MA, Cosar M, Iplikcioglu AC, Ozcan D. Sex steroid and epidermal growth factor profile of giant meningiomas associated with pregnancy. Surg Neurol. 2008; 69(4):356-62; Doi: 10.1016/j.surneu.2007.03.013

6. Mukherjee S, Ghosh SN, Chatterjee U, Chatterjee S. Detection of progesterone receptor and the correlation with Ki-67 labeling index in meningiomas. Neurology India. 2011;59(6):817-22. Doi:10.4103/0028-3886.91357

7. Blitshteyn $\mathrm{S}$, Crook JE, Jaeckle KA. Is there an association between meningioma and hormone replacement therapy? J Clin Oncol. 2008; 26(2): 279-82. Doi:10.1200/JCO.2007.14.2133.

8. Fewings PE, Battersby RD, Timperley WR. Long-term follow up of progesterone receptor status in benign meningioma: a prognostic indicator of recurrence? J Neurosurg. 2000; 2 92(3): doi:10.3171/jns.2000.92.3.0401 
9. Krayenbühl N, Pravdenkova S, Al-Mefty O. De novo versus transformed atypical and anaplastic meningiomas: Comparisons of clinical course, cytogenetics, cytokinetics, and outcome. Neurosurgery 2007. 61(3): 495-503. Doi: 10.1227/01.NEU.0000290895.92695.22

10. Elizabeth B. Claus, Lisa Calvocoressi, Mellisa L, Wrensch M, Wiemels JL, Schildkraut JM. Exogenous hormone use, reproductive factors, and risk of intracranial meningioma in females. J Neurosurg. 2013; 118(3): 649-56. doi: 10.3171/2012.9. JNS12811

11. Ikeri NZ, Anunobi CC, Bankole OB. Progesterone receptor expression and Ki-67 labelling index of meningiomas in the Lagos university teaching hospital. Niger Postgrad Med J. 2018 ;25(1):17-20. doi: 10.4103/npmj.npmj_16_18

12. Touat M, Lombardi G, Farina P, Kalamarides M, Sanson M. Successful treatment of multiple intracranial meningiomas with the antiprogesteronereceptor agent mifepristone (RU486). Acta neurochirurgica. 2014; 156(10):1831-5. doi: 10.1007/s00701-014-2188-4

13. Louis DN, Perry A, Reifenberger G. Von Deimling A, Figarella-Branger D, Cavenee WK. The 2016 World Health Organization Classification of Tumors of the Central Nervous System: a summary. Acta Neuropathol. 2016 ;131(6):803-20. doi: 10.1007/s00401-016-1545-1

14. Zouaoui S, Darlix A, Rigau V, Mathieu-Daude H, Bauchet F, Bessaoud F, et al. Descriptive epidemiology of 13,038 newly diagnosed and histologically confirmed meningiomas in France: 2006-2010. Neuro-Chirurgie. 2018; 64(1): 15-21. doi: 10.1016/j.neuchi.2014.11.013.
15. Kshettry VR, Ostrom QT, Kruchko C, Al-Mefty O, Barnett $\mathrm{GH}$, Barnholtz-Sloan JS. Descriptive epidemiology of World Health Organization grades II and III intracranial meningiomas in the United States. Neuro-Oncol. 2015; 17(8):1166-73. doi: 10.1093/neuonc/nov069

16. Iplikcioglu AC, Hatiboglu MA, Ozek E, Ozcan D. Is progesteron receptor status really a prognostic factor for intracranial meningiomas? Clin Neurol Neurosurg. 2014; 124: 119-22. doi: 10.1016/j.clineuro.2014.06.015

17. Whittle IR, Foo MS, Besser M, Vanderfield GK. Progesterone and oestrogen receptors in meningiomas: biochemical and clinicopathological considerations. Aust $\mathrm{N}$ Z J Surg.1984; 54(4):325-30 PMID: 6593026

18. Perrot-Applanat $M$, Groyer-Picard MT, Kujas $M$. Immunocytochemical study of progesterone receptor in human meningioma. Acta neurochirurgica. 1992;115(12):20-30. PMID: 1595392

19. Mukhopadhyay $M$, Das $C$, Kumari $M$, Sen A, Mukhopadhyay B, Mukhopadhyay B. Spectrum of meningioma with special reference to prognostic utility of ER, PR and Ki67 expression. J Lab Physicians 2017;9(4):308-13 doi: 10.4103/JLP.JLP_158_16. 